\title{
Magnetic properties of oxide-coated iron nanoparticles synthesized by electrodeposition
}

\author{
S. Banerjee ${ }^{\mathrm{a}}, \mathrm{S}$. Roy $^{\mathrm{b}, 1}$, J.W. Chen ${ }^{\mathrm{b}}$, D. Chakravorty ${ }^{\mathrm{a}, *, 2}$ \\ ${ }^{a}$ Indian Association for the Cultivation of Science, Jadavpur, Raja S.C. Mullick Road, Jadavpur, Calcutta 700 032, India \\ ${ }^{\mathrm{b}}$ Department of Physics, National Taiwan University, Taipei, 10617 Taiwan, ROC
}

Received 24 November 1999; received in revised form 5 May 2000

\begin{abstract}
Nanometre-sized iron particles with diameters in the range 4.9-11.1 $\mathrm{nm}$ have been grown within a silica gel matrix by electrodeposition. Electron diffraction pattern shows the presence of an oxide (either $\mathrm{Fe}_{3} \mathrm{O}_{4}$ or $\gamma-\mathrm{Fe}_{2} \mathrm{O}_{3}$ ) shell on these particles. The variation of magnetization as a function of temperature has been analyzed using the Bloch equation. The Bloch exponent obtained as a function of particle size shows a trend different from that predicted theoretically. Zero field cooled (ZFC) and field cooled (FC) measurements on the nanocomposites have been carried out over the temperature range $2-300 \mathrm{~K}$. Coercivity shows a decrease with increasing temperature. Also, a loop shift as a function of temperature is exhibited by the specimens. This shift disappears at a temperature around $50 \mathrm{~K}$. This behavior is ascribed to an exchange interaction of the iron core with a ferrimagnetic oxide (either $\mathrm{Fe}_{3} \mathrm{O}_{4}$ or $\gamma-\mathrm{Fe}_{2} \mathrm{O}_{3}$ ) shell. (C) 2000 Elsevier Science B.V. All rights reserved.
\end{abstract}

Keywords: Iron nanoparticles; Oxide shell; Loop shift

\section{Introduction}

Magnetic behavior of nanoparticles of both ferromagnetic and ferrimagnetic materials has attracted considerable attention in recent years [1-4]. Such interest emanates both from new physics expected from these systems as well as their possible applications in high-density magnetic recording

\footnotetext{
*Corresponding author. Tel.: + 91-33-4734971; fax: + 9133-4732805.

E-mail address: mlsdc@mahendra.iacs.res.in (D. Chakravorty).

${ }^{1}$ On lien from Datar Switchgear Ltd., Nasik, India.

${ }^{2}$ Also affiliated to Jawaharlal Nehru Centre for Advanced Research, Bangalore 560064.
}

media [5]. When the dimension of the magnetic particles falls below a critical value they become single domain and as a consequence exhibit phenomena like superparamagnetism [6], quantum tunnelling of magnetization [7], enhanced coercivities [8] and giant magnetoresistance [9].

A number of techniques have been developed to synthesize magnetic nanoparticles. Some of these are the colloidal chemical method [10,11], highpressure sputtering [12], inert gas condensation [13], layer deposition [14], mechanical attrition [15], and the solvated-metal-atom dispersion method [16]. One of the intriguing features of these particles is the observed reduction in saturation magnetization Ms [17]. An interaction between the surface spins and those in the core has been used to 
explain this behavior [18,19]. More recent investigations have concentrated on the properties of heterogeneous systems consisting of a ferromagnetic metallic core and an antiferromagnetic oxide shell [20]. The importance of the exchange anisotropy [21] in such a situation has been brought out in these studies.

We have recently used an electrodeposition technique to prepare nanosized metallic particles and studied the fractal growth mechanism associated with the process $[22,23]$. This method was used to grow nanoparticles of $\alpha$-Fe in a silica gel medium. The electron diffraction pattern of these composites indicated that the iron particles were coated with a shell of $\mathrm{Fe}_{3} \mathrm{O}_{4}$. The system was therefore thought to be suitable for investigating the interaction at the interface of nanoparticles of iron and $\mathrm{Fe}_{3} \mathrm{O}_{4}$. Magnetic properties of these materials were studied and the results are reported in this paper.

\section{Experiment}

Gels with target compositions $x \mathrm{FeCl}_{3}$, $(100-x) \mathrm{SiO}_{2}(\mathrm{~mol} \%)$ with $x=10,15$ and 40 were prepared by the following procedure. A measured amount of $\mathrm{FeCl}_{3}$ was dissolved in distilled water and ethyl alcohol and the solution was stirred in a magnetic stirrer. Another solution was prepared of tetraethylorthosilicate in a volume of distilled water and ethyl alcohol which was half that taken in the previous one. The two solutions were mixed and one drop of conc. $\mathrm{HCl}$ was added to the resultant solution. The $\mathrm{pH}$ of the latter was found to be $\sim 5$. The solution was stirred for $3 \mathrm{~h}$ when a transparent sol was obtained. The sol was kept in a flat-bottomed petri-dish in ordinary atmosphere for two weeks for gelation. Gel pieces of size $\sim 5 \mathrm{~mm} \times 5 \mathrm{~mm} \times 1 \mathrm{~mm}$ were obtained. These were crushed in a mortar to a size of $\sim 5 \mu \mathrm{m}$.

Electrodeposition was carried out by using a polished iron cathode having dimensions $2.5 \mathrm{~cm} \times 2.5 \mathrm{~cm} \times 1.0 \mathrm{~mm}$ and an iron anode with dimensions $3.0 \mathrm{~cm} \times 1.0 \mathrm{~cm} \times 1.0 \mathrm{~mm}$. A paste was prepared by mixing $10 \mathrm{mg}$ of the gel powder synthesized as above with $0.2 \mathrm{~cm}^{3}$ of distilled water. The cathode surface was coated with the paste. Electrodeposition at voltages 10 and 20, respective- ly, was carried out after placing the anode on the surface of the gel paste. The voltage was kept on for a typical duration of $20 \mathrm{~s}$. The value of deposition current increased from a few microamperes to approximately $1 \mathrm{~A}$ indicating the formation of metallic channels within the gel. The metal formation within the entire gel mass was completed by changing the anode position on the gel paste. On completion of the process, the gel turned black in colour. The gel was dried in a flow of hot air at $333 \mathrm{~K}$ for a few minutes. The gel powder was scraped off the cathode surface. The resulting powder was kept in a vacuum chamber at $373 \mathrm{~K}$ for $30 \mathrm{~h}$.

The microstructures of these powders were studied by a JEM 200CX transmission electron microscope. Details of specimen preparation have been described earlier [24].

Magnetic measurements at room temperature were carried out in a VSM supplied by Digital Measurement 880. Measurements below room temperature were performed in a commercial SQUID magnetometer over the temperature range $2-300 \mathrm{~K}$ with an applied magnetic field up to $12 \mathrm{kOe}$.

\section{Results and discussion}

Fig. 1(a) shows the transmission electron micrograph for a specimen with $10 \mathrm{~mol} \% \mathrm{FeCl}_{3}$ subjected to an electrodeposition voltage of $10 \mathrm{~V}$. Fig. 1 (b) is the corresponding electron diffraction pattern. These are typical of all the samples in the present system. The values of interplanar spacings $d_{h k l}$ were calculated from the diameters of the rings shown in Fig. 1(b). In Table 1 the experimental $d_{h k l}$ values are compared with standard data for $\alpha-\mathrm{Fe}$. It is evident that the particles are composed of iron. Some of the lines indicate the presence of either $\mathrm{Fe}_{3} \mathrm{O}_{4}$ or $\gamma-\mathrm{Fe}_{2} \mathrm{O}_{3}$. Also, the presence of $\alpha-\mathrm{Fe}_{2} \mathrm{O}_{3}$ cannot be ruled out. It is difficult to differentiate between these phases because their lattice parameters are nearly identical. This should be apparent from the standard ASTM data for all these phases as listed in Table 1. We have not considered the presence of $\alpha-\mathrm{Fe}_{2} \mathrm{O}_{3}$ in our subsequent discussions because this does not show any super exchange interaction. In any case, it is evident 
that the iron particles are surrounded by a shell of oxide, either $\mathrm{Fe}_{3} \mathrm{O}_{4}$ or $\gamma-\mathrm{Fe}_{2} \mathrm{O}_{3}$. The oxide shell formation is believed to arise due to an interaction of iron nanoparticles with $\mathrm{H}_{2} \mathrm{O}$ present in the gel
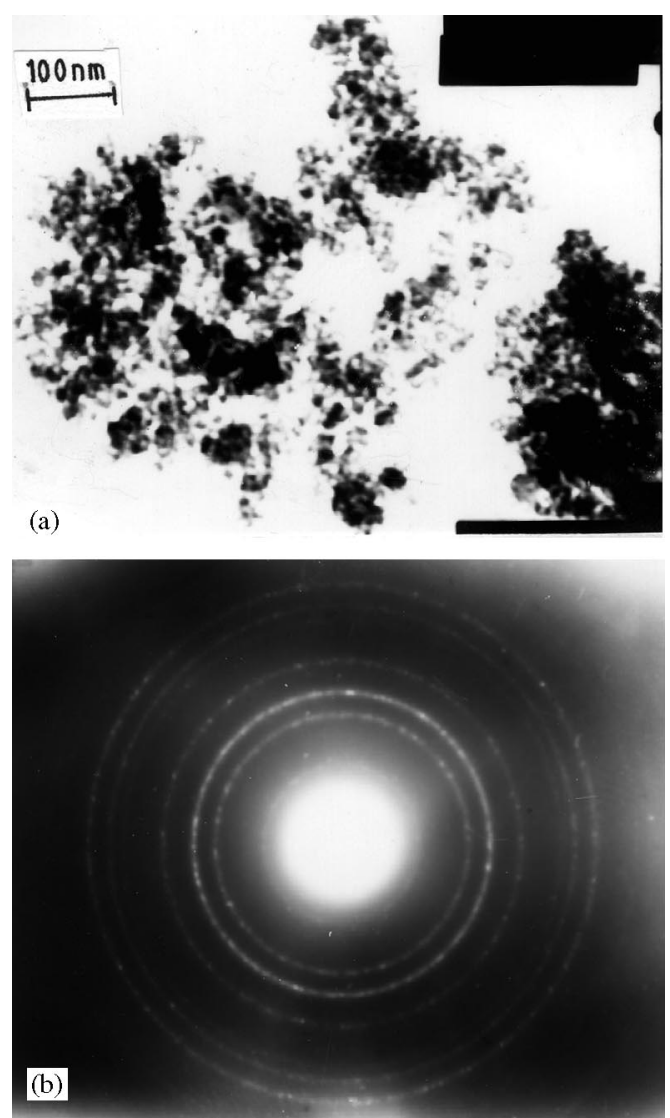

Fig. 1. (a) Transmission electron micrograph for specimen no. 4. (b) Electron diffraction pattern for Fig. 1(a). paste. Fig. 2 is the histogram of iron particles as obtained from Fig. 1(a). The experimental data were fitted to a log-normal distribution function [25]. Table 2 summarizes the values of median diameter $\bar{x}$ and the geometric standard deviation $\sigma$, as extracted from the log-normal fitting for different samples. It is evident from these results that the metal particle size can be reduced by increasing either the metal ion concentration or the voltage applied for electrodeposition. This is consistent with the conclusion drawn from our earlier investigations [22].

Fig. 3 shows the magnetization $(M)$ vs. magnetic field $(H)$ hysteresis curve for specimen no. 4 at

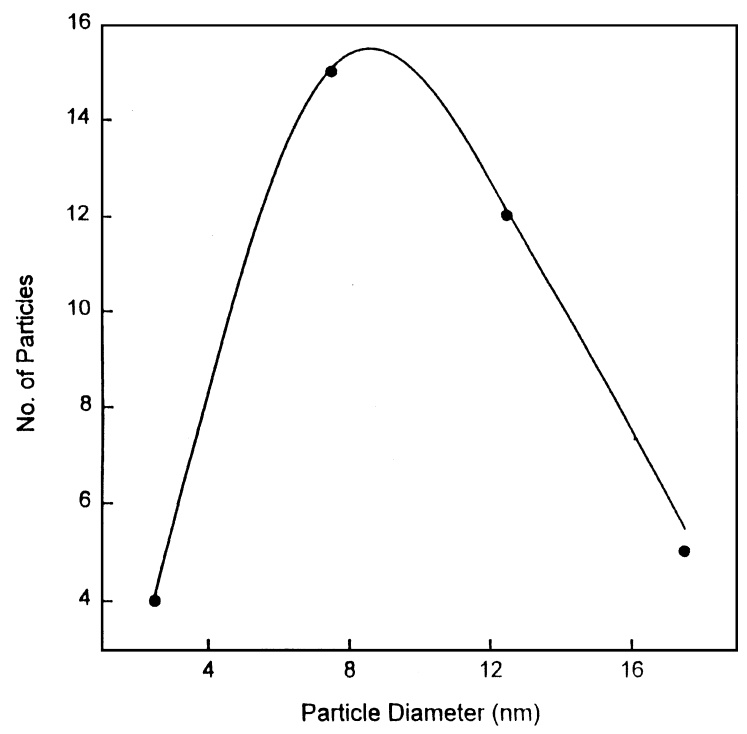

Fig. 2. Histogram of iron particles obtained from Fig. 1(a).

Table 1

Comparison of interplanar spacings for specimen $10 \mathrm{FeCl}_{3}-90 \mathrm{SiO}_{2}\left(\mathrm{~mol}_{\%}\right)$ subjected to $10 \mathrm{~V}$ electrodeposition treatment with standard ASTM data $[31]^{\mathrm{a}}$

\begin{tabular}{|c|c|c|c|c|}
\hline Observed $d_{h k l}(\mathrm{~nm})$ & Standard $\alpha-\mathrm{Fe}(\mathrm{nm})$ & Standard $\mathrm{Fe}_{3} \mathrm{O}_{4}(\mathrm{~nm})$ & Standard $\alpha-\mathrm{Fe}_{2} \mathrm{O}_{3}(\mathrm{~nm})$ & Standard $\alpha-\mathrm{Fe}_{2} \mathrm{O}_{3}(\mathrm{~nm})$ \\
\hline 0.203 & $0.20266(110)$ & & & \\
\hline 0.174 & & $0.1712(422)$ & $0.170(422)$ & $0.169(116)$ \\
\hline 0.144 & $0.14332(200)$ & $0.1483(440)$ & $0.148(440)$ & $0.1452(300)$ \\
\hline 0.118 & $0.11702(211)$ & $0.12112(444)$ & & $0.1189(128)$ \\
\hline 0.110 & & $0.1092(533,731)$ & $0.109(553,731)$ & $0.1102(226)$ \\
\hline 0.101 & $0.10134(220)$ & $0.10489(800)$ & $0.104(652,740)$ & \\
\hline 0.091 & $0.09064(310)$ & $0.08794(931)$ & & $0.0908(310)$ \\
\hline
\end{tabular}

\footnotetext{
${ }^{\text {a }}$ Figures within parentheses in columns 2-5 indicate the $(h k l)$ values for the planes concerned.
} 
Table 2

Summary of median diameter $\bar{x}$ and geometric standard deviation $\sigma$ for different specimens

\begin{tabular}{lllll}
\hline Specimen no. & Composition & $\begin{array}{l}\text { Electrodeposition } \\
\text { voltage }(\mathrm{V})\end{array}$ & $\begin{array}{l}\text { Median diameter } \bar{x} \\
(\mathrm{~nm})\end{array}$ & $\begin{array}{l}\text { Geometric standard } \\
\text { deviation } \sigma\end{array}$ \\
\hline 1 & $40 \mathrm{FeCl}_{3} \cdot 60 \cdot \mathrm{SiO}_{2}$ & 10 & 4.9 & 1.6 \\
2 & $15 \mathrm{FeCl}_{3} \cdot 85 \cdot \mathrm{SiO}_{2}$ & 20 & 5.5 & 1.6 \\
3 & $10 \mathrm{FeCl}_{3} \cdot 90 \cdot \mathrm{SiO}_{2}$ & 20 & 9.5 & 1.4 \\
4 & $10 \mathrm{FeCl}_{3} \cdot 90 \cdot \mathrm{SiO}_{2}$ & 10 & 11.1 & 1.6 \\
\hline
\end{tabular}

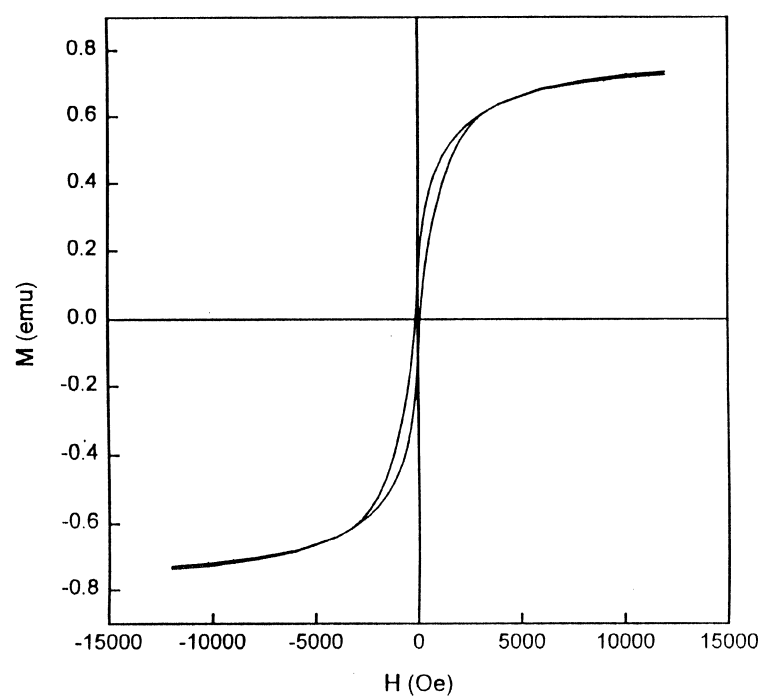

Fig. 3. Magnetization vs. magnetic field hysteresis loop for specimen no. 4 at $300 \mathrm{~K}$.

$300 \mathrm{~K}$. The coercive field $H_{\mathrm{c}}$ is $117 \mathrm{Oe}$ - much larger than that for bulk iron. Table 3 summarizes the values of $H_{\mathrm{c}}$ for the different specimens at $300 \mathrm{~K}$. The values of $H_{\mathrm{c}}$, in general, in all the samples are higher than that for bulk iron. Such large values arise due to the fact that the particle size of $\alpha-\mathrm{Fe}$ is of the order of domain size [26]. The increase of $H_{\mathrm{c}}$ from 48.6 to $117.0 \mathrm{Oe}$ as the iron particle size is increased from 4.9 to $11.1 \mathrm{~nm}$ can be explained as arising due to the reduction of the superparamagnetic effect as will be evident from the following discussion. For ferromagnetic particles of nanometer dimension and having no interaction between them the coercivity $H_{\mathrm{c}}$ is given by [27]

$H_{\mathrm{c}}=H_{\mathrm{co}}\left[1-\left(T / T_{\mathrm{B}}\right)^{1 / 2}\right]$,
Table 3

Values of coercivity $H_{\mathrm{c}}$ for different specimens at $300 \mathrm{~K}$

\begin{tabular}{lll}
\hline Specimen no. & $\begin{array}{l}\text { Mean diameter } \\
\bar{x}(\mathrm{~nm})\end{array}$ & $\begin{array}{l}\text { Coercivity } \\
H_{\mathrm{c}}(\mathrm{Oe})\end{array}$ \\
\hline 1 & 4.9 & 48.6 \\
2 & 5.5 & 62.8 \\
3 & 9.5 & 74.1 \\
4 & 11.1 & 117.0 \\
\hline
\end{tabular}

where $H_{\text {co }}$ is the coercivity at $T=\mathrm{OK}, T$ the temperature of measurement and $T_{\mathrm{B}}$ the blocking temperature above which superparamagnetism sets in. $T_{\mathrm{B}}$ is given by

$T_{\mathrm{B}}=\frac{K^{\prime} V}{25 k}$,

where $K^{\prime}$ is the effective anisotropy constant, $V$ the volume of the ferromagnetic particle and $k$ the Boltzmann constant. An increase in the value of $V$ should also increase $T_{\mathrm{B}}$. From Eq. (1) therefore it follows that the value of $H_{\mathrm{c}}$ should show an increase as the particle size becomes larger. This is consistent with the observed results shown in Table 3.

The variation of saturation magnetization $M$ as a function of temperature for specimen no. 4 is shown in Fig. 4 . The $M$ values were determined by the extrapolation of $H^{-1} \rightarrow 0$. Fig. 5 gives the depression of saturation magnetization relative to its value at $T=\mathrm{OK}$ as a function of temperature. It is evident from this figure that the variation can be represented by [3]

$M(T)=M(0)\left[1-B T^{b}\right]$,

where $B$ is the Bloch constant and $b$ is the Bloch exponent. Such behavior has been ascribed to 


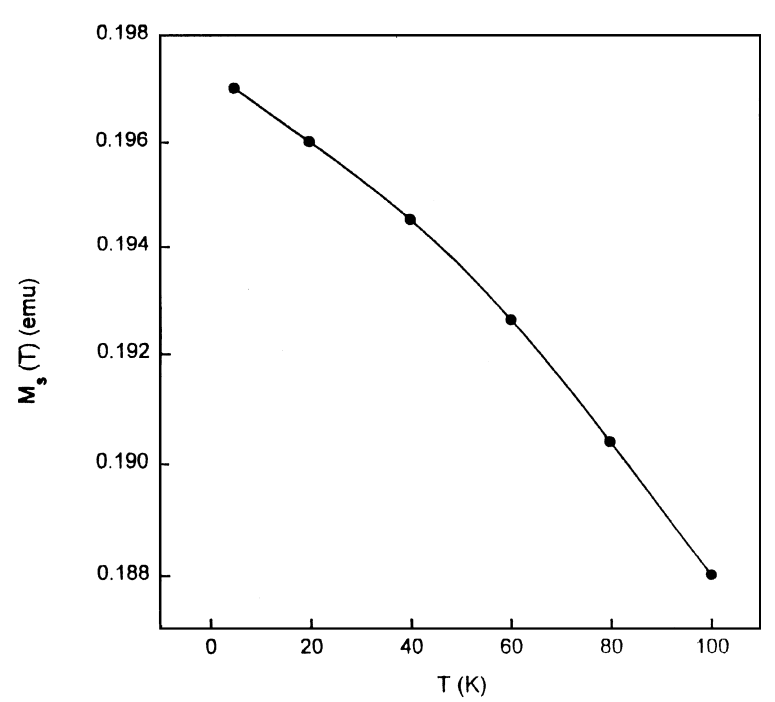

Fig. 4. Variation of saturation magnetization as a function of temperature for specimen no. 4.

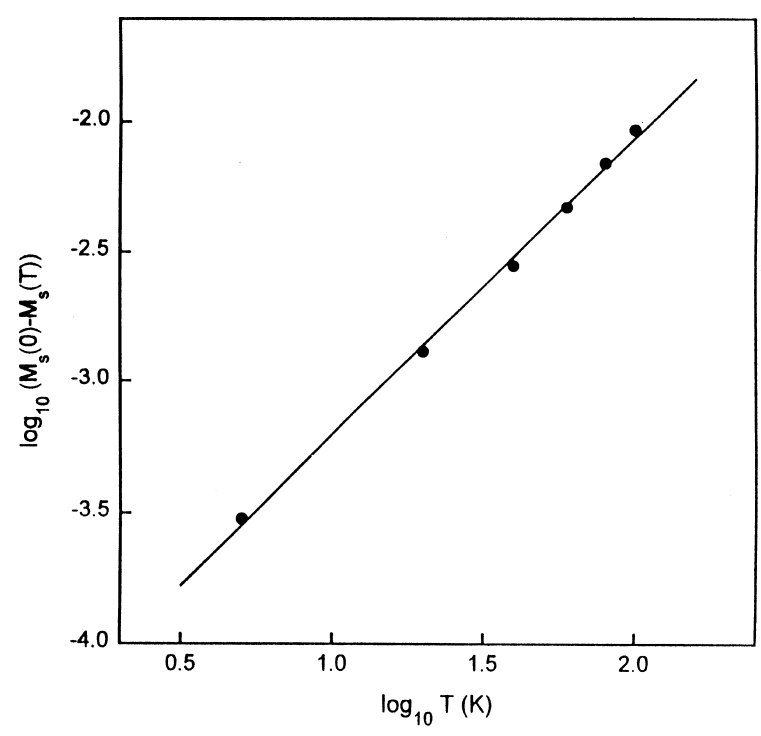

Fig. 5. Saturation magnetization depression relative to its value at $T=\mathrm{OK}$ as a function of temperature for specimen no. 4 .

spin-wave fluctuations [28] at temperatures well below the Curie temperature. We have analyzed the magnetization data for specimens 1 and 4 by fitting the results to Eq. (3). The values of $b$ are found to be 0.79 and 1.14, respectively. These values are less

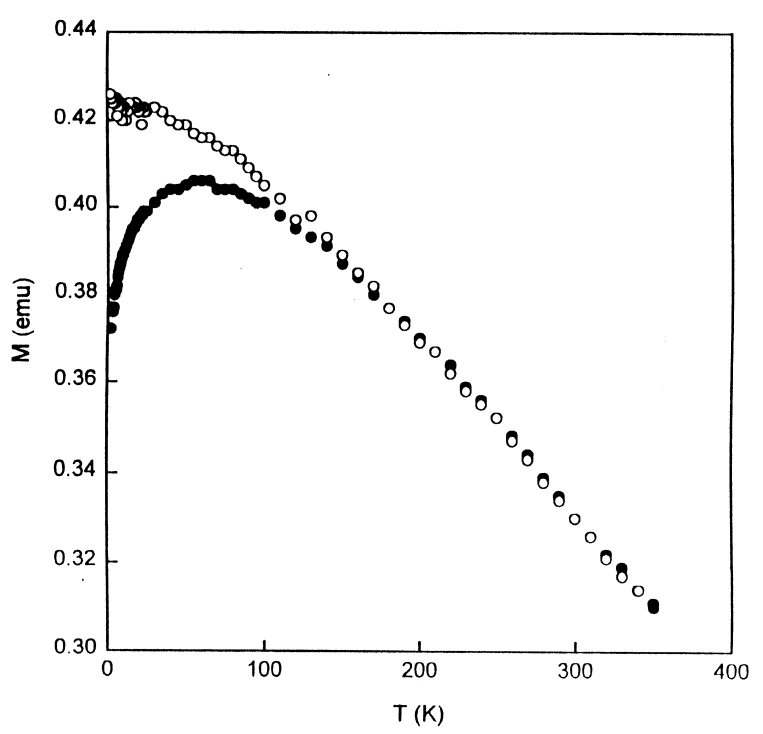

Fig. 6. Variation of magnetization for specimen no. 4 in ZFC and $\mathrm{FC}$ states as a function of temperature. Measuring field: 2 kOe. (O) ZFC, (O) FC.

than that predicted for a bulk sample [3]. Theoretical work has shown that the Bloch exponent $b$ should increase as the particle size is reduced [29]. Our results show an opposite trend but appear to be consistent with those reported earlier in the case of nanosized iron particles [3]. This behavior is believed to be due to the presence of an oxide shell on the iron particles and the interfacial effect resulting from there.

In Fig. 6 is shown the variation of magnetization for specimen no. 4 in both zero field cooled (ZFC) and field cooled (FC) states, respectively, as a function of temperature. The magnetic field used for the FC experiment was $2 \mathrm{kOe}$. A rather high field value was chosen because it was expected that in the present system of core (ferromagnetic) shell (ferrimagnetic) structure a magnetic unidirectional anisotropy (sometimes referred to as exchange anisotropy) could be observed [21]. It is evident from the results that the FC and ZFC states show magnetizations which have identical values at temperatures equal to or higher than $58 \mathrm{~K}$.

Figs. 7(a) and (b) show the hysteresis loops measured at a temperature of $5 \mathrm{~K}$ under the field cooled (FC) and zero field cooled (ZFC) conditions, 


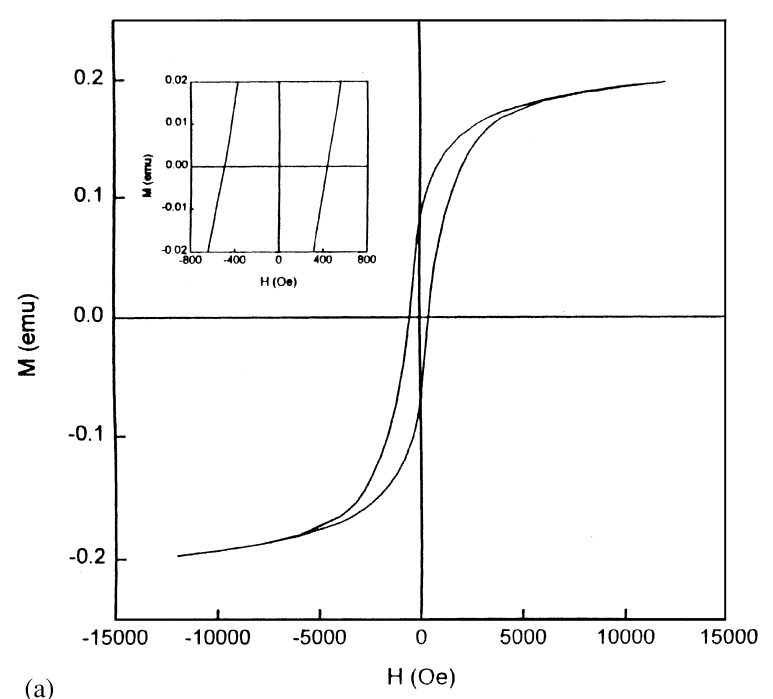

(a)

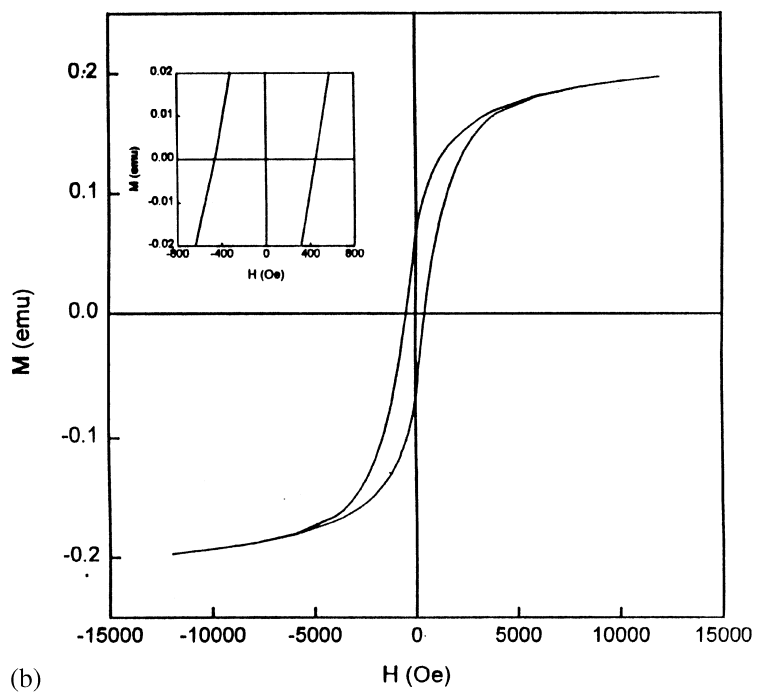

(b)

Fig. 7. (a) Magnetization vs. field loop measured at $5 \mathrm{~K}$ under FC condition for specimen no. 4. (b) Magnetization vs. field loop measured at $5 \mathrm{~K}$ under $\mathrm{ZFC}$ condition for specimen no. 4.

respectively, for specimen no. 4. It is evident that the $\mathrm{ZFC}$ sample displays a hysteresis loop which is symmetric about the origin whereas the FC sample shows a shift of the loop. These measurements were repeated at different temperatures following the same procedure. In Fig. 8, the coercivity $\left(H_{\mathrm{c}}\right)$ of the $\mathrm{ZFC}$ loops is shown as a function of temperature. Fig. 9 gives the variation of loop shift as a function of temperature. In these measurements for the hys-

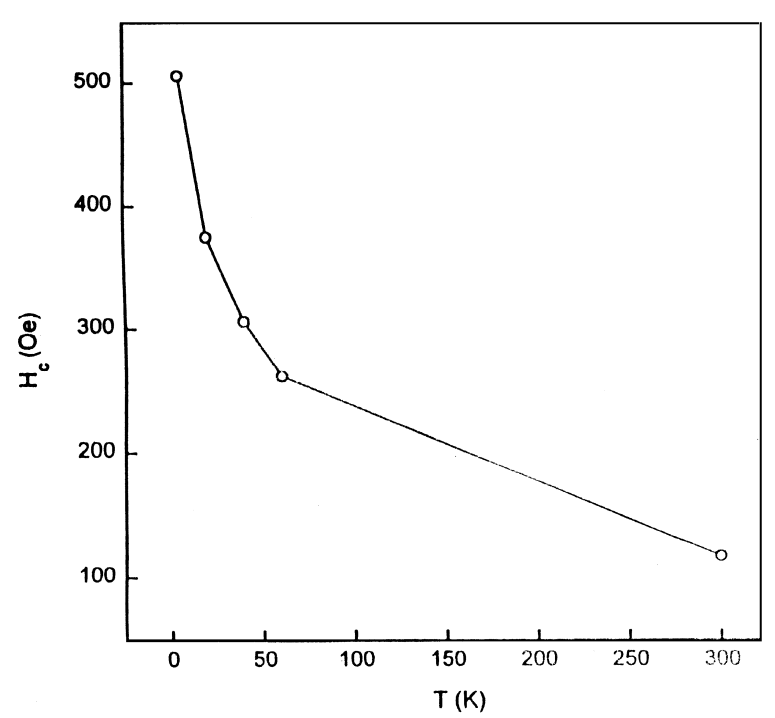

Fig. 8. Variation of coercivity $H_{\mathrm{c}}$ of ZFC loops for specimen no. 4 as a function of temperature.

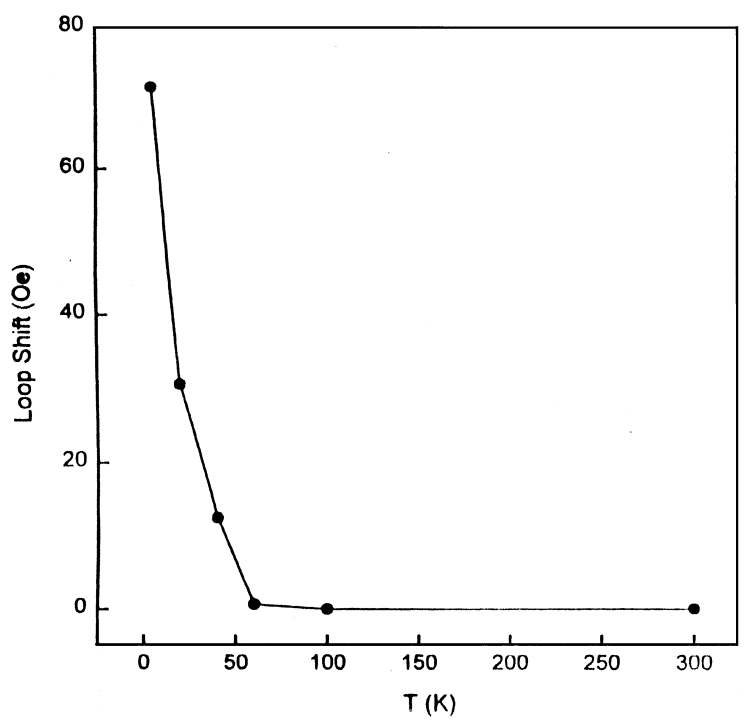

Fig. 9. Variation of loop shift as a function of temperature for specimen no. 4.

teresis loops, a maximum field of $12 \mathrm{kOe}$ was used. This high field was used to ensure that the loop shift could be observed without any uncertainty. This is rationalized in the following manner. As will be discussed later, the loop shift is determined by an exchange interaction between the ferromagnetic 
core of the nanosized iron particles and the oxide surface with spin glass-like magnetic behavior and a freezing temperature $\sim 58 \mathrm{~K}$. Field application at $T>58 \mathrm{~K}$ forces the magnetization of the core to lie along the field direction. Due to the exchange coupling with the core spins, the spins at the oxide layer assume a preferential orientation which becomes frozen below $\sim 58 \mathrm{~K}$. In turn, below this temperature the surface frozen spins will favor the ferromagnetic core of the particle being magnetized in the field cooling direction giving rise to the loop shift observed in our experiments.

The strong temperature dependence of coercivity is believed to arise due to the fact that the frozen surface constitutes a strong hindrance to the reversal of magnetic moment of the ferromagnetic phase. Also, owing to the small size of the iron core some particles may be superparamagnetic at room temperature which would result in a further decrease of $H_{\mathrm{c}}$. The latter model is confirmed by carrying out a ZFC-FC magnetization experiment as a function of temperature at an applied field $H=10 \mathrm{Oe}$. It is to be noted here that the sample was not $\mathrm{AC}$ demagnetized at $300 \mathrm{~K}$ before zero field cooling. Though the latter procedure should have been strictly followed, the presence of superparamagnetic particles as revealed by the experimental results already helped randomize the spins of the nanoparticles. The experimental results also showed that the effective blocking temperature was a little above $300 \mathrm{~K}$. The results are shown in Fig. 10. It is to be noted that the weak field condition of field cooling and the subsequent measurement thereof would give information about the possible superparamagnetic behaviour of the iron cores. It is seen from this figure that the ZFC magnetization does not show a defined maximum and $\mathrm{ZFC}$ and FC branches of the experimental data remain non-overlapping up to values beyond room temperature $(300 \mathrm{~K})$. This behavior is compatible with a broad distribution of core sizes which consequently start exhibiting a superparamagnetic regime at different temperatures.

The loop shift as summarized in Fig. 9 arises due to the presence of the oxide shell around the iron core. The exchange interaction between the ferrimagnetic oxide (either $\mathrm{Fe}_{3} \mathrm{O}_{4}$ or $\gamma-\mathrm{Fe}_{2} \mathrm{O}_{3}$ ) and the $\mathrm{Fe}$ core results in the well-known effect of

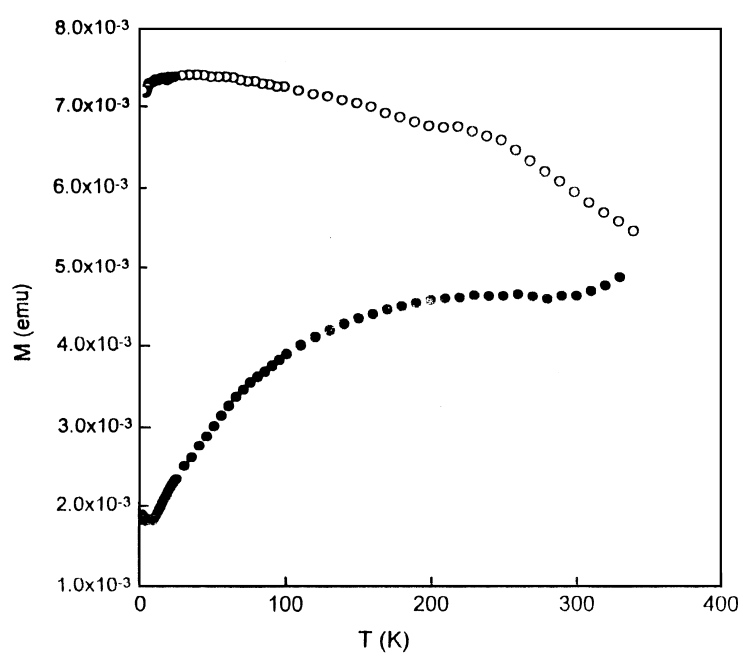

Fig. 10. ZFC and FC magnetization as a function of temperature at an applied field $H=10$ Oe for specimen no. 4. (O) ZFC, (O) FC.

exchange anisotropy [21]. This causes a loop shift as well as an enhanced coercivity. It is to be noted here that ferrimagnetism of the oxide shell (either $\mathrm{Fe}_{3} \mathrm{O}_{4}$ or $\gamma-\mathrm{Fe}_{2} \mathrm{O}_{3}$ ) arises due to an antiferromagnetic interaction between the two cation sub-lattices of the compound concerned. In several studies in recent years it has been proposed that in ferrite nanoparticles a surface spin disorder takes place $[1,4,30]$. This has been corroborated by numerical calculations. The antiferromagnetic superexchange interaction is disrupted at the surface because of missing oxygen ions or other impurity molecules present. Such broken exchange bonds induce surface spin disorder. The onset of loop shift and the separation between $\mathrm{ZFC}$ and FC magnetization curves at about $50 \mathrm{~K}$ are ascribed to a 'freezing' of disordered surface spins of the composite particles. The relationship between the uniaxial anisotropy $K_{\mathrm{u}}$ and the particle volume $V$ is given by [4]

$K_{\mathrm{u}}=\frac{25 k T_{\mathrm{B}}}{V}$.

Using Eq. (4) the value of $K_{\mathrm{u}}$ is estimated to be $2.3 \times 10^{6} \mathrm{ergs} / \mathrm{cm}^{3}$ and $1.6 \times 10^{6} \mathrm{ergs} / \mathrm{cm}^{3}$ for specimens 3 and 4, respectively. It is seen that the value of $K_{\mathrm{u}}$ decreases as the particle diameter is increased 
indicating that the exchange interaction between the core and the shell decreases as the average particle diameter is increased.

In summary, iron particles with a shell of an oxide phase (either $\mathrm{Fe}_{3} \mathrm{O}_{4}$ or $\gamma-\mathrm{Fe}_{2} \mathrm{O}_{3}$ ) have been grown within a silica gel matrix using an electrodeposition method. The particle diameters range from 4.9 to $11.1 \mathrm{~nm}$. ZFC and FC measurements have been carried out over the temperature range $2-300 \mathrm{~K}$. The depression of magnetization as a function of temperature indicates the occurrence of spin-wave fluctuation in iron core. A loop shift is observed for these specimens as a function of temperature. This is ascribed to an exchange interaction between the ferromagnetic core of the nanoparticles and the oxide surface with disordered spins.

\section{Acknowledgements}

D. Chakravorty acknowledges support by the Department of Science and Technology, Govt. of India through Grant no. SP/S2/MO8/95. J.W. Chen acknowledges support through research Grant no. NSC 88-2112-M-002-021 by National Science Council, ROC.

\section{References}

[1] R.H. Kodama, A.E. Berkowitz, E.J. McNiff Jr., S. Foner, Phys. Rev. Lett. 77 (1996) 394.

[2] B. Martinez, X. Obradors, Ll. Balcells, A. Rouanet, C. Monty, Phys. Rev. Lett. 80 (1998) 181.

[3] D. Zhang, K.J. Klabunde, C.M. Sorensen, G.C. Hadijipanayis, Phys. Rev. B 58 (1998) 14167.

[4] R.H. Kodama, A.S. Edelstein, J. Appl. Phys. 85 (1999) 4316.

[5] F.T. Parker, F.F. Spada, T.J. Cox, A.E. Berkowitz, J. Magn. Magn. Mater. 162 (1996) 122.
[6] C. Kittel, Phys. Rev. 70 (1946) 965.

[7] E.M. Chudnovsky, L. Gunther, Phys. Rev. Lett. 60 (1988) 661.

[8] E.F. Kneller, F.E. Luborsky, J. Appl. Phys. 34 (1963) 656.

[9] A.E. Berkowitz, J.R. Mitchell, M.J. Carey, A.P. Young, S. Zhang, F.E. Spada, F.T. Parker, A. Hutten, G. Thomas, Phys. Rev. Lett. 68 (1992) 3745.

[10] J.P. Chen et al., Phys. Rev. B 51 (1995) 11527.

[11] J.A. Becker et al., J. Chem. Phys. 103 (1995) 2520.

[12] A.S. Edelstein et al., Studies of Magnetic Properties of Fine Particles, Elsevier Science, New York, 1992, p. 47.

[13] H. Gleiter, Prog. Mater. Sci. 33 (1989) 223.

[14] S.S. Parkin, N. More, K.P. Roche, Phys. Rev. Lett. 64 (1990) 2304.

[15] C.C. Koch, Nanostructured Mater. 9 (1997) 13.

[16] D. Zhang, G. Glavee, K.J. Klabunde, G.C. Hadjipanayis, C.M. Sorensen, High Temp. Mater. Sci. 36 (1996) 93.

[17] A.E. Berkowitz, W.J. Shuele, P.J. Flanders, J. Appl. Phys. 39 (1968) 1261.

[18] A.H. Morrish, K. Hanada, P.J. Schurer, J. Phys. C 37 (1976) 6.

[19] T. Okada, H. Sekizawa, F. Ambe, T. Yamada, J. Magn. Magn. Mater. 31-34 (1983) 105.

[20] C. Prados, M. Multigner, A. Hernando, J.C. Sanchez, A. Fernandez, C.F. Conde, A. Conde, J. Appl. Phys. 85 (1999) 6118.

[21] W.H. Meiklejohn, C.P. Bean, Phys. Rev. 105 (1957) 904.

[22] S. Banerjee, D. Chakravorty, Appl. Phys. Lett. 72 (1998) 1027.

[23] Sourish Banerjee, Soomit Banerjee, Anindya Datta, Dipankar Chakravorty, Europhys. Lett. 46 (1999) 346.

[24] A.K. Maity, D. Nath, D. Chakravorty, J. Phys.: Condens. Matter 8 (1996) 5717.

[25] B. Roy, D. Chakravorty, J. Phys: Condens. Matter 2 (1990) 9323.

[26] S.H. Liou, C.L. Chien, Appl. Phys. Lett. 52 (1988) 512.

[27] E.H. Frei, S. Shtrikman, D. Treves, Phys. Rev. 106 (1957) 446.

[28] R. Kubo, T. Nagamiya, Solid State Physics, McGraw-Hill, New York, 1968, p. 580.

[29] P.V. Hendriksen, S. Linderoth, P.V. Lindgard, Phys. Rev. B 48 (1993) 7259.

[30] L. Del Bianco, A. Hernando, M. MuHigner, C. Prados, J.C. Sanchoz-Lopez, A. Fernandez, C.F. Conde, A. Conde, J. Appl. Phys. 24 (1998) 2189.

[31] Power Diffraction File, American Society for Testing and Materials, 1972, pp. 6-15. 